\title{
Development and validation of a salt knowledge questionnaire
}

\author{
Rani Sarmugam ${ }^{1, *}$, Anthony Worsley ${ }^{1}$ and Vicki Flood ${ }^{2}$ \\ ${ }^{1}$ School of Exercise and Nutrition Sciences, Deakin University, 221 Burwood Highway, Burwood, VIC 3125, \\ Australia: ${ }^{2}$ School of Health Sciences, University of Wollongong, Wollongong, NSW, Australia
}

Submitted 21 May 2012: Final revision received 25 October 2012: Accepted 30 January 2013: First published online 18 March 2013

\begin{abstract}
Objective: Initiatives promoting the reduction of high-salt food consumption by consumers need to be partly based on current levels of salt knowledge in the population. However, to date there is no validated salt knowledge questionnaire that could be used to assess population knowledge about dietary salt (i.e. salt knowledge). Therefore, the aim of the present study was to develop and validate a salt knowledge questionnaire.

Design: A cross-sectional study was conducted on an online web survey platform using convenience, snowball sampling. The survey questionnaire was evaluated for content and face validity before being administered to the respondents.

Setting: Online survey.

Subjects: A total of forty-one nutrition experts, thirty-two nutrition students and thirty-six lay people participated in the study.

Results: Item analyses were performed to evaluate the psychometric properties of the test items. Twenty-five items were retained to form the final set of questions. The total scores of the experts were higher than those of the students and lay people $(P<0 \cdot 05)$. The total salt knowledge score showed significant correlations with use of salt at the table $(\rho=-0 \cdot 197, P<0 \cdot 05)$ and inspection of the salt content in food products when shopping $(\rho=0 \cdot 400 ; P<0 \cdot 01)$.

Conclusions: The questionnaire demonstrated sufficient evidence of construct validity and internal consistencies between the items. It is likely to be a useful tool for the evaluation and measurement of levels of salt knowledge in the general population.
\end{abstract}

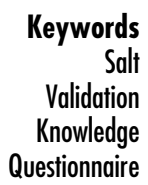

Dietary sodium intake has been identified as one of the major risk factors for high blood pressure and $\mathrm{CVD}^{(1-3)}$. Cost-benefit analyses have demonstrated that reduction of dietary salt is a cost-effective measure to reduce the disease burden associated with $\mathrm{CVD}^{(4-6)}$.

Although more than $75 \%$ of dietary salt comes from processed foods ${ }^{(7)}$, consumer awareness and education is important to empower consumers to choose products with lower salt content and reduce the amount of discretionary salt intake. Consumer understanding and evaluation of salt knowledge, attitude and behaviours has been recommended as one of the key components of a successful salt reduction programme ${ }^{(8,9)}$.

To date, several studies have been conducted to assess the levels of salt knowledge in the population ${ }^{(10-14)}$. While there were some common elements in the questions used across these studies, no attempt has been made to validate them, i.e. to establish whether, in fact, they assess consumers' knowledge. The use of unreliable, unvalidated questionnaires to measure nutrition knowledge has been suggested as being responsible for the inconsistencies observed in the relationships between knowledge and dietary behaviours ${ }^{(15,16)}$.
Given the importance of consumer knowledge as a fundamental aspect of population monitoring and providing information to policy makers and stakeholders involved in salt reduction initiatives ${ }^{(8)}$, we believe there is an urgent need for a validated questionnaire to assess consumer salt knowledge and enable comparisons between different salt reduction communication programmes. Therefore, the aim of the present study was to describe the development and validation of a salt knowledge questionnaire in an Australian adult population.

\section{Method}

\section{Study design}

A cross-sectional study was conducted on an online web survey platform using convenience, snowball sampling ${ }^{(17)}$.

\section{Participants}

The study population consisted of experts (dietitians/ nutritionists), dietetics or nutrition students and lay people. Experts were recruited via an email which was sent to a list of dietitians and nutritionists in Australia. Students were 
recruited through lectures attended by Dietetics and Nutrition students in two universities. Lay people were recruited through the professional and informal social networks of the researchers. In addition, the study was advertised in website forums. The invitation email also requested the potential participants to forward the email to others who might be interested in taking part in the study.

\section{Procedure}

The participants were invited to answer a self-administered online questionnaire ${ }^{(18)}$, which could be completed at their convenience within 20 to $40 \mathrm{~min}$. The survey was kept open for four months.

\section{The questionnaire}

The first part of the questionnaire required participants to indicate which group they belonged to, i.e. nutritionist/ dietitians, or nutrition/dietetics students, or lay people. Next, they were asked to rate their overall knowledge of dietary salt based on a four-point scale which ranged from 'very high' to 'low' before they proceeded to the main part of the questionnaire.

The questionnaire consisted of four parts: (i) salt knowledge; (ii) beliefs about salt; (iii) dietary behaviours related to salt intake; and (iv) demographic information.

\section{Salt knowledge}

Questions. The salt knowledge section assessed two main domains of knowledge: (i) declarative knowledge, i.e. awareness of things and processes (i.e. the properties of nutrients such as salt and foods); and (ii) procedural knowledge or 'know how knowledge, ${ }^{\text {(16) }}$. The declarative knowledge section included questions drawn from the literature relating to the identification of diet-disease associations, expert recommendations and sources of high-salt foods ${ }^{(15)}$, as well as common misconceptions about salt and health (e.g. 'Cutting down salt may cause leg cramps', 'Drinking more water can neutralise salt in my diet'). Where possible, items were derived from previous studies on salt knowledge ${ }^{(10,12-14,19)}$, to enable comparison of results with past studies ${ }^{(15)}$.

The procedural knowledge section related to purchasing behaviours, eating at home and eating out. These items were based on health education messages targeted at healthy populations ${ }^{(20-22)}$.

A total of seventy-three questions was developed and reviewed by a nutritionist (V.F.) and a behavioural scientist (A.W.). These experts reviewed all of the questions to ensure they clearly represented the knowledge domains intended to be measured (content validity). Their inputs were taken into consideration in the construction of the items and the response formats ${ }^{(23)}$. The questionnaire was also pre-tested among five lay individuals for comprehension and question format (face validity). After the evaluation, sixty-five items were retained to form the questionnaire. These items were evaluated for psychometric criteria (discrimination index, item difficulty index, item-to-total correlation; see Statistical analysis). This resulted in a final set of twenty-five questions (fifteen questions which met all of the psychometric criteria and ten questions which were included to maintain content validity ${ }^{(15)}$; Table 2 ).

Scoring. The questions were presented to the respondents in the form of multiple-choice and true or false response scales (Table 2). All correct responses for multiple-choice items were scored as 1 , while incorrect responses which included 'don't know' or 'not sure' and non-responses were assigned a score of 0 .

True or false questions were presented using fivepoint response scales: 'certainly true', 'probably true', 'not sure', 'probably wrong' and 'certainly wrong'. In order to differentiate between lack of knowledge and knowledge held with low levels of confidence ${ }^{(24)}$, a score of 2 was assigned for 'certainly true', 1 for 'probably true' and 0 for incorrect answers which included 'not sure' and non-responses. Negative statements were reversed prior to scoring.

The salt knowledge items were summed to yield scores for each subset of questions corresponding to dietary recommendations, diet-disease relationships, perceived salt content of commonly eaten foods, common misconceptions and label reading habits.

In addition, a total salt knowledge score was derived by summing the twenty-five items that were included in the final version of the questionnaire (Table 2).

\section{Beliefs related to salt}

While there are distinct epistemological differences in the definitions of knowledge and beliefs ${ }^{(24,25)}$, both affect behaviour in the same way. For example, the Theory of Planned Behaviour posits that beliefs held by a person influence his/her attitudes which in turn determine his/ her behaviour ${ }^{(26)}$. This is because the thinking processes and motivational effects of an individual holding a belief (regardless of the truth) act in similar ways ${ }^{(24)}$.

The belief items were based on items used in past studies $^{(12)}$ and themes derived from websites and web discussions about salt ${ }^{(20,27)}$. Beliefs related to salt were assessed using four items: (i) 'My health would improve if I lowered the amount of salt in my diet'; (ii) 'In general, salt-free food tastes bad'; (iii) 'Salt makes food tasty'; and (iv) 'Salt should be used in cooking to enhance the taste of the food'. These belief items were measured on five-point Likert scales ranging from 'certainly wrong' to 'certainly true'. Principal component analysis showed that the second, third and fourth items formed one factor (Cronbach's $\alpha=0.58$ ). These three items were retained and were summed to derive a total beliefs score about the importance of the taste of salt. Higher scores indicate stronger beliefs about the importance of the taste of salt. 
Behaviours associated with higher salt intake

Dietary behaviour. Dietary behaviours associated with salt consumption were assessed using a list of items which included: (i) the use of discretionary salt, which contributes about $20 \%$ of the salt in Australians' diets (aged 2 years and older) ${ }^{(28)}$; (ii) dietary practices relating to the use of salt during meal preparation, e.g. fresh ingredients and substitution of salt with herbs and spices as recommended in the Dietary Guidelines for Australia ${ }^{(22)}$; (iii) consumption of fast food and sauces ${ }^{(29,30)}$; and (iv) the consumption of salted snacks.

Participants were asked to indicate how frequently they engaged in the particular behaviours. Responses ranged from never or not applicable to 1-3 times/week, 4-6 times/week and 7 times/week.

Food shopping behaviour. Frequencies of food shopping behaviours (e.g. 'Looked for the salt content in food products when shopping', 'Purchased a product labelled "low salt" or "reduced salt") were assessed using five-point scales. Response options ranged from never to 4 or more times/week.

Scores were assigned according to the frequencies (1 for never, 2 for 2-3 times/week, etc.). Higher scores indicated higher frequency in engaging in particular behaviours.

\section{Demographic information}

Demographic information including age, gender and highest level of education was elicited from the respondents.

\section{Statistical analysis}

Data analysis was conducted using the statistical software package IBM SPSS Statistics for Windows version 20•0. Descriptive statistics were calculated to describe the study sample. Item analyses were conducted by calculating: (i) the item difficulty index (IDI), the proportion of individuals who answered an item correctly ${ }^{(31)}$; (ii) the discrimination index (DI), which was used to determine whether each item was capable of discriminating between the highest and lowest scorers ${ }^{(31)}$; and (iii) itemto-total correlations (ITC), which measure the extent each item shares the same domain or common core as the other items ${ }^{(32)}$. The acceptable range of IDI for the present study was set as $0 \cdot 3$ to $0 \cdot 9$, the minimal DI was set at $0 \cdot 2^{(31)}$ and ITC at $0 \cdot 30^{(33)}$

The construct validity of the questionnaire was established by comparing the responses of the three groups of individuals who were expected to have different levels of nutrition knowledge based on the degree of their nutritional training ${ }^{(15)}$, i.e. dietitians/nutritionists should have greater knowledge than nutrition/dietetics students who in turn should have greater knowledge than lay people. The construct validity of the questionnaire was evaluated using the Kruskal-Wallis $H$ test. If any significant differences were observed in the total salt knowledge scores and each subset of questions (dietary recommendations, diet-disease relationships, perceived salt content of commonly eaten foods, common misconceptions and label reading) between the three groups (i.e. experts, students and lay people), the Mann-Whitney $U$ test was used to evaluate pair-wise differences (i.e. experts $v$. students, experts $v$. lay people).

Spearman's rho correlation coefficient was used to examine the associations between the total salt knowledge and total beliefs scores and the set of dietary behaviours associated with high salt consumption.

\section{Results}

\section{Description of sample}

Table 1 shows the demographic characteristics of the study participants. A total of 133 individuals accessed the survey website. Of these, twenty-four provided incomplete responses and were excluded from the data analysis. The remaining 109 provided usable responses for the final analyses. Forty-one respondents $(37 \cdot 6 \%)$ were experts (dietitians/nutritionists), thirty-two (29.4\%) were dietetics/ nutrition students and thirty-six $(33 \cdot 0 \%)$ were lay people. The overwhelming majority of the participants were female $(93 \cdot 1 \%)$. More than $50 \%$ of the respondents were the main food shopper or food preparer for the household. The majority $(73 \cdot 1 \%)$ of the nutrition experts rated their overall salt knowledge as high or very high, while the majority of nutrition students (65.6\%) rated their knowledge as medium. A third of the lay people rated their knowledge on dietary salt as low and slightly more than $50 \%$ rated their knowledge as medium.

\section{Psychometric properties of the salt knowledge items}

Table 2 shows the items that were included in the final set of questions. Twenty-one out of the twenty-five items had an acceptable level of item difficulty (IDI $=0 \cdot 3-0 \cdot 9$ ), sixteen of the items had DI values above $0 \cdot 2$ (which is generally considered sufficient to discriminate between good and poor performers) and sixteen met the criterion set for ITC $(\geq 0 \cdot 30)$.

\section{Construct validity}

The group differences in sub-scores and total salt knowledge score are shown in Table 3. The total salt knowledge score for all participants ranged from 9 to 30 (maximum possible score was 31) with a mean of 20.39 (sD 5•08). The mean total salt knowledge score and subscores were significantly and consistently higher among the experts, followed by the students (all $P<0 \cdot 05$ ).

\section{Relationship between total salt knowledge and belief scores with dietary behaviours}

There were significant inverse correlations between total salt knowledge score and frequent use of salt at the table 
Table 1 Demographic characteristics of the study sample: respondents to the online salt knowledge questionnaire, Australia

\begin{tabular}{|c|c|c|c|}
\hline & $\begin{array}{l}\text { Dietitians/nutritionists } \\
\text { (n 41)† }\end{array}$ & $\begin{array}{l}\text { Dietetics/nutrition students } \\
\text { (n 32)† }\end{array}$ & $\begin{array}{l}\text { Lay people } \\
\qquad(n 36) t\end{array}$ \\
\hline & $\%$ & $\%$ & $\%$ \\
\hline \multicolumn{4}{|l|}{ Gender } \\
\hline Male & $5 \cdot 1$ & $3 \cdot 2$ & $12 \cdot 9$ \\
\hline Female & $94 \cdot 9$ & $96 \cdot 8$ & $87 \cdot 1$ \\
\hline \multicolumn{4}{|l|}{ Age (years) } \\
\hline$<20$ & $0 \cdot 0$ & $33 \cdot 3$ & $3 \cdot 2$ \\
\hline $20-30$ & $41 \cdot 0$ & $63 \cdot 3$ & $19 \cdot 4$ \\
\hline $31-40$ & $25 \cdot 6$ & $3 \cdot 3$ & $22 \cdot 6$ \\
\hline $41-50$ & $12 \cdot 8$ & $0 \cdot 0$ & $22 \cdot 6$ \\
\hline $51-60$ & $12 \cdot 8$ & $0 \cdot 0$ & $19 \cdot 4$ \\
\hline$>60$ & $7 \cdot 7$ & $0 \cdot 0$ & $12 \cdot 9$ \\
\hline \multicolumn{4}{|l|}{ Country of birth } \\
\hline Australian & $71 \cdot 1$ & $77 \cdot 4$ & $83 \cdot 3$ \\
\hline Others & $28 \cdot 9$ & $22 \cdot 6$ & $16 \cdot 7$ \\
\hline \multicolumn{4}{|l|}{ Highest level of education } \\
\hline Left school at 18 or earlier & $0 \cdot 0$ & $45 \cdot 2$ & $19 \cdot 4$ \\
\hline TAFE or college diploma, certificate or formal trade qualification & $0 \cdot 0$ & $16 \cdot 1$ & $16 \cdot 1$ \\
\hline Bachelor degree/graduate diploma/graduate certificate & $43 \cdot 2$ & $35 \cdot 5$ & $38 \cdot 7$ \\
\hline Postgraduate degree & $56 \cdot 8$ & $3 \cdot 2$ & $25 \cdot 8$ \\
\hline \multicolumn{4}{|l|}{ Cooking for the household } \\
\hline Main role & $71 \cdot 8$ & $41 \cdot 9$ & $67 \cdot 7$ \\
\hline Share responsibility & $25 \cdot 6$ & $45 \cdot 2$ & $25 \cdot 8$ \\
\hline \multicolumn{4}{|l|}{ Food shopping for the household } \\
\hline Main role & $74 \cdot 4$ & $45 \cdot 2$ & $45 \cdot 2$ \\
\hline Share responsibility & $23 \cdot 1$ & $35 \cdot 5$ & $45 \cdot 2$ \\
\hline \multicolumn{4}{|l|}{ Self-rated knowledge of dietary salt } \\
\hline Very high & $14 \cdot 6$ & $0 \cdot 0$ & $2 \cdot 8$ \\
\hline High & $58 \cdot 5$ & $34 \cdot 4$ & $13 \cdot 9$ \\
\hline Medium & $26 \cdot 8$ & $65 \cdot 6$ & $52 \cdot 8$ \\
\hline Low & $0 \cdot 0$ & 0.0 & $30 \cdot 6$ \\
\hline
\end{tabular}

TAFE, technical and further education.

tTotal for each row may vary slightly due to missing data.

$(\rho=-0 \cdot 197, \quad P<0 \cdot 05)$ and consumption of fast food $(\rho=-0 \cdot 293, P<0 \cdot 01$; Table 4). The total salt knowledge score was also correlated positively with healthier dietary behaviours associated with lower use of salt, such as using fresh ingredients and herbs and spices in cooking $(\rho=0 \cdot 327, P<0 \cdot 01)$ and looking for salt content in food products when purchasing foods $(\rho=0 \cdot 400, P<0 \cdot 01)$. However, there was no significant correlation between the use of salt in cooking and salt knowledge.

Significant correlations were also observed between beliefs about the importance of the taste of salt and the use of table salt $(\rho=0 \cdot 401, P<0 \cdot 01)$, salt in cooking $(\rho=0 \cdot 443$, $P<0 \cdot 01)$, table sauces $(\rho=0 \cdot 207, P<0 \cdot 05)$ and frequent consumption of salted snacks $(\rho=0 \cdot 391, P<0 \cdot 01)$.

\section{Discussion}

The final twenty-five-item questionnaire demonstrated adequate construct validity and good internal reliability.

The psychometric analysis of the items showed that their discriminatory properties varied between sections. For example, items in the dietary recommendations section, which require 'technical knowledge', had higher discriminatory values; i.e. there were more distinct differences between the experts and the lay people. All four items relating to dietary recommendations demonstrated good item discrimination (DI $>0 \cdot 2$ ) and three of the four questions were answered correctly by less than half of the respondents. The lay persons' responses observed in this section are similar to those seen in other studies which used similar questions ${ }^{(11,12,14,34)}$.

In contrast, three out of five questions about the health risks associated with higher salt intake were answered correctly by at least $90 \%$ of the respondents (IDI $\geq 0 \cdot 90$ ), hence their lower discriminatory values $(\mathrm{DI}<0 \cdot 2)$. Past studies have also shown that over $80 \%$ of consumers possessed greater levels of awareness of the health risks associated with salt intake $e^{(11-14)}$.

As in other studies of nutrition knowledge and dietary behaviours ${ }^{(35,36)}$, the correlations observed between total salt knowledge score and dietary behaviours associated with high-salt food consumption were low or moderate. This may be because knowledge often acts as an indirect predictor of behaviour through mediating variables such as attitudes and intention ${ }^{(35)}$.

Slightly stronger correlations were observed between beliefs about the importance of the taste of salt and use of salt at the table and in cooking, suggesting that taste preference may also may play an important part in 
Table 2 Item analyses for each item in the online salt knowledge questionnaire

\begin{tabular}{|c|c|c|c|c|}
\hline Category & Section/question & DI & IDI & ITC \\
\hline \multirow{27}{*}{ Declarative knowledge } & Dietary recommendations & & & \\
\hline & Which of the following statements best describes the relationship between salt and sodium? (salt contains sodium)t & 0.5 & $0 \cdot 7$ & 0.422 \\
\hline & How many grams of salt is equivalent to one teaspoon of salt? $(4 \mathrm{~g})$ & $0 \cdot 6$ & 0.5 & 0.387 \\
\hline & A product is considered as 'low in salt' when it contains... (120/100 mg) & $0 \cdot 9$ & 0.5 & 0.643 \\
\hline & What is the maximum recommended daily amount of salt for an adult in Australia? $(6 \mathrm{~g}) \ddagger$ & $0 \cdot 3$ & 0.2 & 0.269 \\
\hline & Conditions which might be associated with high salt intakes & & & \\
\hline & High blood pressure & $0 \cdot 0$ & $1 \cdot 0$ & - \\
\hline & High blood sugar & $0 \cdot 4$ & 0.7 & 0.320 \\
\hline & Strokeł & $0 \cdot 0$ & $1 \cdot 0$ & 0.000 \\
\hline & Kidney diseaseł & $0 \cdot 1$ & 0.9 & 0.095 \\
\hline & Osteoporosis & 0.5 & $0 \cdot 4$ & 0.391 \\
\hline & Salt content of commonly eaten foods & & & \\
\hline & White bread (medium) $\ddagger$ & $-0 \cdot 1$ & 0.5 & -0.087 \\
\hline & Bacon (high) $\ddagger$ & 0.2 & 0.9 & 0.337 \\
\hline & White rice (boiled) (low) & $0 \cdot 6$ & 0.8 & 0.425 \\
\hline & Beef steak (uncooked) (low) & 0.5 & $0 \cdot 7$ & 0.357 \\
\hline & Mix vegetables (frozen) (low) & 0.5 & $0 \cdot 8$ & $0 \cdot 420$ \\
\hline & Corn flakes (medium)‡ & $-0 \cdot 1$ & 0.4 & -0.075 \\
\hline & Vegemite (high) & $0 \cdot 3$ & $0 \cdot 8$ & 0.325 \\
\hline & Cheddar cheese (processed) (high) & $0 \cdot 4$ & $0 \cdot 8$ & 0.430 \\
\hline & Salt is naturally present in fresh food $\neq, \S$ & $0 \cdot 2$ & $0 \cdot 7$ & $0 \cdot 139$ \\
\hline & Fast foods are high in saltt, ,§ & $0 \cdot 0$ & $1 \cdot 0$ & $0 \cdot 150$ \\
\hline & Bread is one of the main sources of salt in Australians' diets $\S$ & 0.5 & $0 \cdot 6$ & 0.435 \\
\hline & Common misconceptions & & & \\
\hline & Sea salt is better than table saltll,§ & $0 \cdot 7$ & $0 \cdot 6$ & 0.554 \\
\hline & Drinking more water can neutralise salt in my dietll,§ & $0 \cdot 4$ & $0 \cdot 8$ & 0.434 \\
\hline & Cutting down on salt causes leg cramps\|,§ & 0.5 & 0.8 & 0.388 \\
\hline \multirow[t]{3}{*}{ Procedural knowledge } & Label reading & & & \\
\hline & Which pasta sauce has the highest salt content & $0 \cdot 2$ & 0.9 & $0 \cdot 192$ \\
\hline & If you see a TICK logo on a packet of breakfast cereal, what do you think about the product? & 0.5 & $0 \cdot 8$ & 0.447 \\
\hline
\end{tabular}

DI, discrimination index; IDI, item difficulty index; ITC, item-to-total correlation.

tCorrect answers are in parentheses after each item.

fltems which were retained for the purpose of content validity.

Indicates items were scored as: $2=$ 'certainly true', $1=$ 'probably true', $0=$ all others; otherwise items were scored as $1=$ correct answer; $0=$ all others. Negative statements were reversed prior to scoring.

illtems were reversed coded prior to scoring. 
Table 3 Mean and range of scores for each group of respondents to the online salt knowledge questionnaire

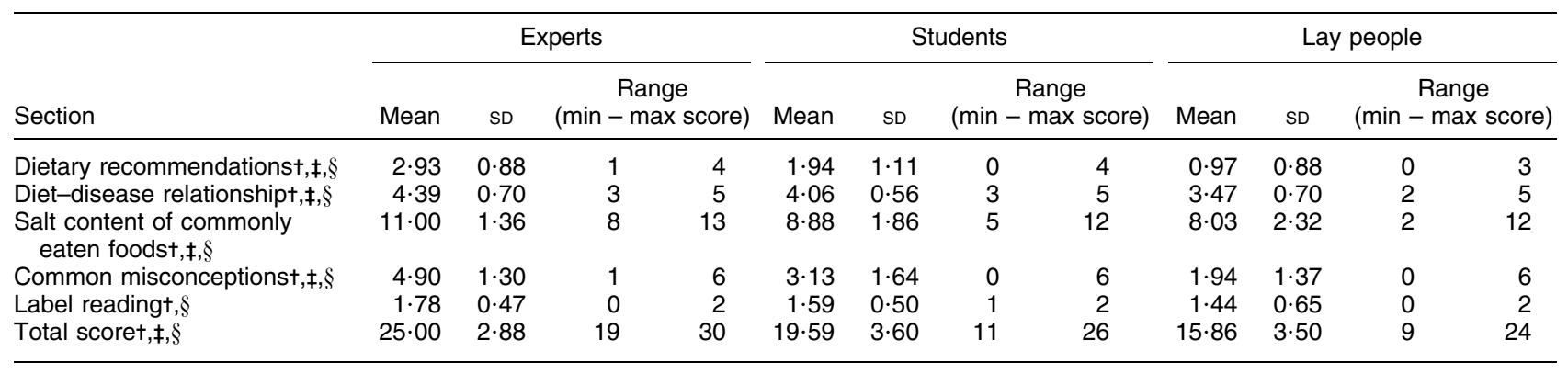

tSignificantly different between the three groups (Kruskal-Wallis $H$ test, $P<0.05$ ).

$\ddagger$ Significantly different, experts $V$. students (Mann-Whitney $U$ test, $P<0.05$ ).

$\S$ Significantly different, experts $v$. lay people (Mann-Whitney $U$ test, $P<0.05$ ).

Table 4 Correlations of total salt knowledge score and total beliefs related to taste of salt score with self-reported frequencies of dietary behaviours associated with higher salt intake

\begin{tabular}{lcc}
\hline & Total salt knowledge score $(\rho)$ & Total beliefs score $(\rho)$ \\
\hline Use of table salt & $-0 \cdot 197^{\star}$ & $0 \cdot 401^{\star *}$ \\
Added salt during cooking & $-0 \cdot 051$ & $0 \cdot 443^{\star *}$ \\
Cooked meals from scratch/fresh ingredients & $0 \cdot 321^{\star *}$ & $-0 \cdot 067$ \\
Used herbs and spices as flavouring for cooking & $0 \cdot 327^{\star \star}$ & $-0 \cdot 159$ \\
Table sauces (e.g. tomato sauce, chilli sauce, barbeque sauce) & $-0 \cdot 171$ & $0 \cdot 207^{\star}$ \\
Ready-made sauces (e.g. pasta sauces, marinades) for cooking & $-0 \cdot 068$ & $0 \cdot 182$ \\
Frequency of eating fast food & $-0 \cdot 293^{\star *}$ & $0 \cdot 166$ \\
Frequency of eating salted snacks & $-0 \cdot 175$ & $0 \cdot 391^{\star *}$ \\
Looked for the salt content in food products when shopping & $0 \cdot 400^{\star *}$ & $-0 \cdot 108$ \\
\hline
\end{tabular}

Significant correlation: ${ }^{*} P<0.05,{ }^{* *} P<0.01$.

determining dietary behaviour ${ }^{(37)}$. The relationships observed between beliefs and behaviour, especially with the discretionary salt use, could also be due to the specific phrasing of the statements used in the study. For example, the specificity of the belief statement 'Salt should be used in cooking to enhance the taste of the food' is more likely to predict behaviour than a more general belief statement ${ }^{(24)}$.

While we used item analyses to guide the evaluation of each item, we were aware that these analyses reflect only the internal consistency of the items and not the validity of the items ${ }^{(38)}$. As such, some items that had low discriminatory values but were considered essential to measure salt knowledge levels in the population (content validity) were retained ${ }^{(15)}$. The inclusion of these items did not reduce the discriminatory value of the questionnaire since significant differences were observed in the total and sectional salt knowledge scores between the groups (which supports their construct validity).

Several factors such as the sampling method (convenience sampling), lack of information about the characteristics of non-responders and presence of chronic diseases may limit the generalisability of the study findings. Also, as in previous studies ${ }^{(39-41)}$, females were over-represented in our sample as were those with higher levels of education.

Some of the items used in the present study are applicable only for Western diets where the majority of salt in the diet comes from processed foods. In developing countries (e.g. China) where the majority of salt in the diet is added to food during food preparation $^{(42)}$, additional questions on food preparation should be considered. Similarly, the misconceptions and beliefs about salt may vary between cultures ${ }^{(43)}$. Therefore, reassessments of these items are required prior to using the questionnaire in cultures with different salt beliefs and behaviours.

A further limitation of the study concerns the use of self-reported frequencies of dietary behaviours associated with high salt consumption as a proxy of dietary sodium intake. Even though self-reported use of table salt has been found to be correlated with actual behaviour ${ }^{(44)}$ and urinary sodium ${ }^{(45)}$, discretionary salt intake only represents about $20 \%$ of salt intake in the $\operatorname{diet}^{(28)}$. Therefore, future studies should extend the validation of this questionnaire against other established sodium intake measurements such as $24 \mathrm{~h}$ urinary sodium excretion or dietary recalls and test the questionnaire for its test-retest reliability.

\section{Conclusion}

The current questionnaire is likely to be a useful tool for researchers and policy makers who wish to measure levels of salt knowledge in general populations or to 
examine the effectiveness of public education programmes. Further investigation is needed to improve the assessment of procedural knowledge and to test the validity of the questionnaire in other populations.

\section{Acknowledgements}

Sources of funding: The study was supported by the Higher Degree Research Student Funds, University of Wollongong. Ethics: The study was approved by the University of Wollongong Research Ethics Committee. Conflicts of interest: The authors declare no conflict of interest. Authors' contributions: R.S and A.W. were responsible for the design of study. R.S. analysed and wrote the paper with contributions from A.W. V.F. assisted in the review of the questionnaire and manuscript. Acknowledgements: The authors thank Dr Anne-Maree Parrish for her invaluable assistance in the preparation of the ethics application for this project and all of the survey respondents for their participation.

\section{References}

1. Yang Q, Liu T, Kuklina EV et al. (2011) Sodium and potassium intake and mortality among US adults: prospective data from the Third National Health and Nutrition Examination Survey. Arch Intern Med 171, 1183-1191.

2. Cook NR, Obarzanek E, Cutler JA et al. (2009) Joint effects of sodium and potassium intake on subsequent cardiovascular disease: the Trials of Hypertension Prevention followup study. Arch Intern Med 169, 32-40.

3. Strazzullo P, D'Elia L, Kandala N-B et al. (2009) Salt intake, stroke, and cardiovascular disease: meta-analysis of prospective studies. BMJ 339, b4567.

4. Bibbins-Domingo K, Chertow GM, Coxson PG et al. (2010) Projected effect of dietary salt reductions on future cardiovascular disease. $N$ Engl J Med 362, 590-599.

5. Asaria P, Chisholm D, Mathers C et al. (2007) Chronic disease prevention: health effects and financial costs of strategies to reduce salt intake and control tobacco use. Lancet 370, 2044-2053.

6. Palar K \& Sturm R (2009) Potential societal savings from reduced sodium consumption in the US adult population. Am J Health Promot 24, 49-57.

7. Mattes RD \& Donnelly D (1991) Relative contributions of dietary sodium sources. J Am Coll Nutr 10, 383-393.

8. World Health Organization (2010) Strategies to Monitor and Evaluate Population Sodium Consumption and Sources of Sodium in the Diet: Report of a Joint Technical Meeting Convened by $\mathrm{WHO}$ and the Government of Canada, October 2010. Geneva: WHO; available at http://whqlibdoc.who.int/publications/2011/9789241501699_ eng.pdf

9. Legetic B \& Campbell N (2011) Reducing salt intake in the Americas: Pan American Health Organization actions. J Health Commun 16, 37-48.

10. Consensus Action on Salt and Health (2003) Adults Survey 2003. http://www.actiononsalt.org.uk/less/what/adults/ index.html (accessed January 2012).

11. Wyllie A, Moore R, Brown R (2011) Salt Consumer Survey. Wellington: Ministry of Agriculture and Forestry; available at http://www.foodsafety.govt.nz/elibrary/industry/ salt-survey.pdf
12. Grimes CA, Riddell LJ \& Nowson CA (2009) Consumer knowledge and attitudes to salt intake and labelled salt information. Appetite 53, 189-194.

13. Webster JL, Li N, Dunford EK et al. (2010) Consumer awareness and self-reported behaviours related to salt consumption in Australia. Asia Pac J Clin Nutr 19, 550-554.

14. Chariton K, Yeatman H, Houweling F et al. (2010) Urinary sodium excretion, dietary sources of sodium intake and knowledge and practices around salt use in a group of healthy Australian women. Aust N Z J Public Health 34, 356-363.

15. Parmenter K \& Wardle J (1999) Development of a general nutrition knowledge questionnaire for adults. Eur J Clin Nutr 53, 298-308.

16. Worsley A (2002) Nutrition knowledge and food consumption: can nutrition knowledge change food behaviour? Asia Pac J Clin Nutr 11, Suppl. 3, S579-S585.

17. VanderStoep SW \& Johnson DD (2008) Research Methods for Everyday Life: Blending Qualitative and Quantitative Approaches. San Francisco, CA: Jossey-Bass.

18. Dillman DA (2007) Internet, Mail, and Mixed-mode Surveys: The Tailored Design Method, 2nd ed. Hoboken, NJ: John Wiley \& Sons Inc.

19. Marshall S, Bower JA \& Schröder MJA (2007) Consumer understanding of UK salt intake advice. Br Food J 109, 233-245.

20. AWASH, Austalian Division of World Action on Salt \& Health (n.d.) For Consumers. http://www.awash.org.au/ for-consumers/ (accessed March 2010).

21. Food Standards Agency (2010) Salt tips and myth. http:// tna.europarchive.org/20100929190231/http://www.eatwell. gov.uk/healthydiet/fss/salt/salttipsandmyths/ (accessed May 2010).

22. National Health and Medical Research Council (2005) Food for Health. Dietary guidelines for Australians, A guide to healthy eating. http://www.nhmrc.gov.au/_files_nhmrc/ publications/attachments/n31.pdf (accessed March 2010).

23. Parmenter K \& Wardle J (2000) Evaluation and design of nutrition knowledge measures. J Nutr Educ 32, 269-277.

24. Kemm J (1991) Health education and the problem of knowledge. Health Promot Int 6, 291-296.

25. Oxford Dictionaries Online (2012) Oxford reference. http://www.oxfordreference.com/view/10.1093/acref/97801 99534067.001.0001/acref-9780199534067-e-2104 (accessed January 2013).

26. Ajzen I (1991) The theory of planned behavior. Organ Behav Hum Decis Process 50, 179-211.

27. Penberthy D (2010) In defence of salt. http://www.thepunch. com.au/articles/in-defence-of-salt/ (accessed September 2010).

28. Food Standards Australia New Zealand (2011) Sodium and salt. http://www.foodstandards.gov.au/scienceandeducation/ factsheets/factsheets/sodiumandsalt.cfm (accessed September 2012).

29. Food Standards Australia New Zealand (2012) Sodium levels in a range of packaged and take-away foods. http://www.foodstandards.gov.au/scienceandeducation/ publications/sodiumlevelsinarange4648.cfm (accessed March 2012).

30. Dunford E, Webster J, Barzi F et al. (2010) Nutrient content of products served by leading Australian fast food chains. Appetite 55, 484-489.

31. Oosterhof A (2001) Classroom Applications of Educational Measurement, 3rd ed. Upper Saddle River, NJ: Merrill.

32. Churchill GA Jr (1979) A paradigm for developing better measures of marketing constructs. J Mark Res 16, 64-73.

33. Nunnally JC \& Bernstein IH (1994) Construction of conventional test. In Psychometric Theory, 3rd ed., pp. 293-334 [J Vaicunas and JR Belser, editors] New York: McGraw Hill. 
34. Consensus Action on Salt and Health (2011) Adults Survey 2011. http://www.actiononsalt.org.uk/less/what/Salt\%20intake \%20survey\%202011/index.html (accessed March 2012).

35. Sapp SG \& Jensen HH (1997) Reliability and validity of nutrition knowledge and diet-health awareness tests developed from the 1989-1991 diet and health knowledge surveys. J Nutr Educ 29, 63-72.

36. Dickson-Spillmann M \& Siegrist M (2011) Consumers' knowledge of healthy diets and its correlation with dietary behaviour. J Hum Nutr Diet 24, 54-60.

37. Kim GH \& Lee HM (2009) Frequent consumption of certain fast foods may be associated with an enhanced preference for salt taste. J Hum Nutr Diet 22, 475-480.

38. Mehrens WA \& Lehmann IJ (1973) Measurement and Evaluation in Education and Psychology. New York: Holt, Rinehart and Winston, Inc.

39. Hendrie GA, Cox DN \& Coveney J (2008) Validation of the general nutrition knowledge questionnaire in an Australian community sample. Nutr Diet 65, 72-77.
40. Hendrie GA, Coveney J \& Cox D (2008) Exploring nutrition knowledge and the demographic variation in knowledge levels in an Australian community sample. Public Health Nutr 11, 1365-1371.

41. Venter I (2008) Construction of a valid and reliable test to determine knowledge on dietary fat of higher-educated young adults. S Afr J Clin Nutr 21, 133-139.

42. Brown IJ, Tzoulaki I, Candeias V et al. (2009) Salt intakes around the world: implications for public health. Int $J$ Epidemiol 38, 791-813.

43. Smith SL, Quandt SA, Arcury TA et al. (2006) Aging and eating in the rural, southern United States: beliefs about salt and its effect on health. Soc Sci Med 62, 189-198.

44. Mittelmark MB \& Sternberg B (1985) Assessment of salt use at the table: comparison of observed and reported behavior. Am J Public Health 75, 1215-1216.

45. Jeffery P, Nowson C, Riddell L et al. (2012) Salt intake in Victorian adults. Presented at Seminar - World Salt Awareness Week, Melbourne, Victoria, 26 March 2012. 